\title{
Lo Sviluppo della Competenza Comunicativa Orale dell'Italiano come Lingua Straniera. Accenni neurolinguistici e glottodidattici.
}

Martina Brazzolotto

Università di Venezia Ca'Foscari martinabrazzolotto@gmail.com

\begin{abstract}
Le recenti scoperte neurolinguistiche hanno messo in evidenza come avviene la comprensione e la produzione del messaggio orale nel nostro cervello. Queste informazioni dovrebbero essere tenute in considerazione quando si insegna una lingua straniera, per favorire il successo scolastico a tutti gli alunni.
\end{abstract}

Key words: glottodidattica, neurolinguistica, comprensione, produzione, competenza comunicativa.

Resumo: As recentes descobertas neurolinguísticas evidenciaram como se processa a compreensão e a produção da mensagem oral em nosso cérebro. Essas informações deveriam ser levadas em consideração quando se ensina uma língua estrangeira a fim de conduzir todos os alunos ao êxito escolar.

Palavras-chave: Ensino de línguas. Neurolinguística. Compreensão. Produção. Competência comunictiva.

\section{Introduzione}

La glottodidattica è una disciplina che trae le proprie conoscenze da molte scienze, tra cui la neurolinguistica. In questo articolo vorremmo ricavare, dalle recenti scoperte di alcuni studi, che si sono occupati di analizzare i correlati neurali del linguaggio, alcune implicazioni glottodidattiche. In particolare faremo riferimento all'apprendimento della 
competenza comunicativa orale, suddivisa in comprensione e produzione. Consapevoli che questi due processi non sono facilmente divisibili, ma sempre integrati, vorremmo proporre delle indicazioni per i docenti che insegnano la lingua italiana all'estero. Spesso non capiamo perché alcuni alunni non hanno successo o vorremmo fornire loro una tecnica specifica per sviluppare una certa abilità. Il presente articolo fornisce queste informazioni con l'obiettivo di aumentare la qualità dell'insegnamento e dell'apprendimento della lingua straniera.

\section{Il linguaggio orale: nozioni di neuroscienze.}

Nel corso dell'ultimo secolo è emersa una chiara relazione tra il linguaggio e il cervello, recenti studi svelano come tale organo comprende e produce il pensiero attraverso le parole. Prima Paul Broca (1861) e poi Karl Wernicke (1874) si occuparono di studiare soggetti con disturbi di produzione e comprensione di espressioni vocali. Entrambi i neurologi scoprirono che l'emisfero sinistro del cervello umano è dominante in queste due funzioni, anche se collocate in due aree distinte: la prima si troverebbe sul lobo frontale, mentre la seconda sarebbe situata sulla superficie superiore del lobo temporale (BEAR, CONNORS, PARADISO. 2009. pp. 643-644).

Le regioni del cervello coinvolte nel linguaggio orale sono molto più numerose delle semplici aree di Broca e di Wernicke, poiché, grazie alle nuove tecniche di neuroimmagine (tomografia a emissione di positroni (PET) e risonanza magnetica funzionale (fRMI), ne è stata dimostrata l'esistenza. Nella Tabella 1 infatti notiamo come alcuni compiti corrispondano a specifiche aree cerebrali. 


\begin{tabular}{|c|c|}
\hline Compito & Aree cerebrali attivate \\
\hline $\begin{array}{l}\text { Ascolto } \\
\text { parole }\end{array}$ & Corteccia uditiva primaria e secondaria. \\
\hline $\begin{array}{l}\text { Pronuncia di } \\
\text { parole }\end{array}$ & $\begin{array}{l}\text { Corteccia motrice primaria e aree motorie supplementare, } \\
\text { scissura silviana (bilaterale). }\end{array}$ \\
\hline $\begin{array}{l}\text { Generazione di } \\
\text { verbi }\end{array}$ & $\begin{array}{l}\text { Area frontale inferiore sinistra, parte anteriore del giro del } \\
\text { cingolo e nel lobo temporale posteriore. }\end{array}$ \\
\hline
\end{tabular}

Tabella 1: Compiti e rispettive aree cerebrali attivate'.

Ora ci chiediamo se i sistemi cerebrali deputati al linguaggio siano i medesimi sia per la lingua madre sia per la lingua straniera. Per soddisfare questa curiosità, indispensabile per la trattazione del nostro argomento, bisogna rifarsi agli studi che si sono occupati di indagare l'attività neurale di individui afasici poliglotti, che hanno subito una lesione cerebrale. La sostanziale differenza che emerse tra i soggetti che parlavano una sola lingua e quelli che ne usavano abitualmente due o più, riguardava l'età di acquisizione: i bilingui precoci, cioè quelli che avevano acquisito la nuova lingua entro i sette anni, quando utilizzavano la lingua seconda (L2) attivavano le stesse aree cerebrali dei soggetti che parlavano solo la loro madrelingua. Altri studi condotti da Perani et al. (1996, pp. 2439-2344) dimostrarono che l'area di Wernicke (come già detto, specializzata soprattutto per la comprensione del messaggio orale) si attivava in entrambi i soggetti, indipendentemente dall'età di acquisizione. Gli stessi studiosi sostengono che l'utilizzo di reti neurali diverse sia dovuto anche al livello di padronanza e non solo agli anni di un individuo, quindi un adulto che possiede un'ottima conoscenza della L2, metterebbe in atto gli stessi meccanismi cognitivi di un madrelingua. Numerose tomografie infatti, dimostrano che le aree cerebrali deputate

1 Informazioni reperite nel testo di BEAR, Mark F., CONNORS, Barry W., PARADISO, Michael A. Neuroscienze: Esplorando il cervello. Terza edizione. Milano: Elsevier, 2009, pp. 657-660. 
all'apprendimento di una nuova lingua, prima del periodo critico, sono le medesime che si attivano per l'apprendimento della lingua nativa (cfr. Fabbro, 1996). Possiamo affermare quindi che l'apprendimento di una nuova lingua avviene nello stesso modo durante i primi 7 anni di vita (FABBRO 2004, p.18). Interessante infine citare un'altra ricerca degli stessi autori che ha indagato l'attivazione neurale durante l'esposizione della lingua seconda e di una lingua completamente sconosciuta. I dati dimostrano che le aree cerebrali non sono sovrapponibili, il cervello quindi mette in atto ulteriori meccanismi di elaborazione, quando è esposto a suoni completamente estranei.

Quello che ne deriva, come sostengono Ferrari e Palladino (2012, p.35) è che le aree che si attivano differiscono in base all'età, al livello di padronanza e alla distanza linguistica tra L1 e L2, variabili che si devono tenere in considerazione durante l'insegnamento di una nuova lingua. Nei paragrafi che seguono tralasceremo le lingue che sono molto diverse tra loro, poiché prenderemo in esame quelle con la stessa distanza sociolinguistica, cioè con una struttura linguistica, culturale e metodi di insegnamento simili (SANTIPOLO, 2006, pp. 22-23).

\section{La comprensione del messaggio orale}

La comprensione del messaggio orale avviene grazie l'interazione di più aree cerebrali: corteccia uditiva bilaterale, giri temporali superiore, medio e inferiore dell'emisfero sinistro, giro frontale inferiore dell'emisfero sinistro, gangli della base e aree motorie e premotorie (MARINI, 2012, p. 267).

Analizziamo nello specifico come si verifica l'elaborazione delle parole ascoltate mettendo in relazione le informazioni che provengono dalla neurolinguistica con degli esempi concreti di apprendimento dell'italiano come lingua straniera. 
Secondo Marini (2010, p.113) ci sono delle fasi specifiche che si susseguono durante l'ascolto di un enunciato orale:

a) Analisi uditiva. Subito dopo aver ricevuto l'input linguistico, l'ascoltatore distingue i rumori di sottofondo dalle parole e successivamente ha luogo il riconoscimento delle caratteristiche acustiche, che coinvolge la corteccia uditiva bilaterale. Il nostro cervello quindi riesce sempre a individuare la voce umana tra altri stimoli sonori.

b) Trasformazione dello stimolo acustico in formato fonologico. Il passaggio dal suono al riconoscimento del fonema risulta semplice e naturale, quando ascoltiamo la nostra lingua nativa, ma è complesso per uno studente che si accinge a capire parole mai udite prima, con dei fonemi che non appartengono al proprio repertorio linguistico. Se pensiamo alla fonetica italiana, costituita da una forte variabilità di suoni, che cambiano in base alla lettera che precede o segue, deduciamo che l'insegnante dovrebbe occuparsi anche di curare la pronuncia, attraverso una metodologia che corrisponda alla naturale acquisizione, come verrà meglio descritto nel paragrafo 2.1. In questa fase dunque l'ascoltatore è impegnato a determinare la prosodia, cioè "l'insieme degli schemi ritmici e intonativi generati dalle parole" ${ }^{2}$, che differisce da lingua a lingua, con lo scopo di identificare le singole parole. Il più importante strumento prosodico è l'accento che in italiano è libero, cioè non cade sempre sulla stessa sillaba. Questa caratteristica complica l'apprendimento del lessico italiano, perché lo studente dovrà imparare per ogni termine oltre che il significato anche la corretta inflessione di voce.

c) Selezione lessicale. Il riconoscimento dei vocaboli secondo

2 MARINI, 2010. p. 79. 
Liberman e Mattingly (1985, pp. 1-36) avverrebbe inconsapevolmente infatti, la ricezione sarebbe strettamente legata alla produzione che si esplicherebbe per imitazione, ipotesi confermata dalla recente scoperta dei neuroni specchio. Secondo un'altra ipotesi invece, una parola udita viene riconosciuta grazie dei confronti con altre simili, come descritto dal modello della coorte (MARSLEN-WILSON, 1987, pp. 71-102). Le singole espressioni verrebbero raggruppate in base la stessa struttura fonologica e selezionate attraverso una continua esclusione, che si arresta quando la parola ascoltata e quella ipotizzata coincidono. Solitamente l'alta frequenza d'uso e il contesto linguistico ${ }^{3}$ agevolano questo processo, anzi se prendiamo in considerazione quest'ultimo fattore, potremmo affermare che la lingua italiana, essendo strutturata secondo l'ordine SVO (soggetto, verbo, oggetto), potrebbe aiutare a cogliere il significato delle parole.

d) Accesso al lemma e unificazione grammaticale. Riconosciuta la parola, si accede alle informazioni morfosintattiche. L'analisi grammaticale avverrebbe in modo costruttivo e non di recupero, come invece avviene per la fase precedente.

La riflessione linguistica è un processo che ci permette di comprendere il significato globale (scanning) attraverso i collegamenti che ricaviamo dalle parole. Questo meccanismo viene "costruito" in modo consapevole in quanto tutte le lingue possiedono delle eccezioni, e in modo particolare l'italiano. Possiamo prender in considerazione la formazione del genere nei nomi che non è standard, ci sono infatti, molti casi particolari come per esempio il femminile di "eroe" non è

3 Il contesto linguistico nella comunicazione orale si riferisce all'argomento generale che facilita la comprensione delle singole parole. 
"eroa" come verrebbe in automatico se seguissimo la regola, ma "eroina". Lo stesso vale per "cane" che al femminile non è "cana" bensì "cagna".

La comprensione del linguaggio orale dunque avviene a partire dal particolare (analisi dei suoni) per raggiungere gradualmente il significato globale, inizialmente coinvolgendo entrambi gli emisferi e successivamente quello sinistro.

\subsection{Implicazioni glottodidattiche}

In questo paragrafo vorremmo trarre delle implicazioni glottodidattiche che siano congruenti con il naturale processo di comprensione del messaggio orale che avviene nel nostro cervello. Le proposte che inseriremo saranno riferite in particolare agli studenti che possiedono un basso livello di padronanza della lingua straniera.

Le informazioni che ci provengono dalla neurolinguistica ci confermano che le tecniche glottodidattiche, per apprendere una nuova lingua, devono variare in base all'età degli alunni, al livello di padronanza posseduta oltre che alla distanza linguistica. Tenendo in considerazioni queste variabili e partendo dal presupposto che ogni fase del processo di comprensione di un enunciato possa essere sviluppata durante l'apprendimento di una nuova lingua, proponiamo per ognuna alcune indicazioni che l'insegnante di lingua straniera potrà seguire in classe o selezionare in base agli errori che prevalentemente compiono gli alunni.

La prima fase che mettiamo in atto durante la ricezione di un messaggio orale è quella dell'analisi uditiva. Solitamente nel contesto scolastico l'attenzione è orientata prevalentemente nei contenuti o processi da apprendere, quindi il cervello dei nostri alunni distingue facilmente l'input linguistico da altre fonti 


\section{Lo Sviluppo della Competenza Comunicativa Orale dell'Italiano come Lingua Straniera. Accenni neurolinguistici e glottodidattici. Martina Brazzolotto}

sonore.

Nell'apprendimento di una nuova lingua invece, quello che maggiormente ostacola la comprensione riguarda l'individuazione dell'inizio e della fine di una parola, per questo spesso chiediamo al nostro interlocutore di parlare più piano. Alcuni studi di neuroscienze hanno constatato che questo è uno dei problemi che affrontano anche i bambini quando imparano la loro lingua nativa. Secondo Jenny Saffran e i suoi colleghi dell'Università del Wisconsin durante i primi anni di vita le parole vengono apprese secondo una percezione statistica, cioè alcune combinazioni di suoni sarebbero più probabili di altre (BEAR, CONNORS, PARADISO 2009, p. 662). In questa seconda fase (trasformazione dello stimolo acustico in formato fonologico), oltre la funzione demarcativa ${ }^{4}$ bisogna analizzare anche la funzione pragmatica, propria degli aspetti extralinguistici. Per esempio, per ogni espressione orale abbiniamo un'intonazione diversa a seconda che sia una domanda, un'affermazione o un ordine. Quando insegniamo una nuova lingua dobbiamo enfatizzare maggiormente l'altezza e l'intensità del tono di voce, perché anche in questo modo aiutiamo la comprensione del messaggio orale. La prosodia, infine, ci trasmette anche gli aspetti emotivi intrinseci all'enunciato. Se infatti esprimiamo impazienza o sdegno verso gli studenti si potrebbe attivare il filtro affettivo, che inibisce la comprensione (KRASHEN, 1985, p. 3). Il nostro tono di voce quindi, oltre che far capire il significato delle parole, dovrebbe trasmettere tranquillità, per creare un ambiente sereno di apprendimento.

Per comprendere quali pratiche glottodidattiche mettere in atto dobbiamo fare riferimento alla memoria implicita che è specializzata nel riconoscimento e apprendimento fonologico, strettamente collegato alla

4 Per funzione demarcativa intendiamo la capacità di individuare l'inizio e la fine di una parola ascoltata. 
prosodia. Questo tipo di memoria a lungo termine è caratterizzata da causalità, inconsapevolezza automatismo e da progressione, grazie la pratica (CANGIÀ 2011, p. 138; DALOISO 2009, p. 69). L'acquisizione fonologica quindi sarà immagazzinata in modo naturale se si adotta un insegnamento induttivo, per scoperta, dove lo studente può individuare gradualmente le analogie di suoni che presentano alcune parole. Per favorire tale processo si consiglia di pronunciare le parole scandendole lentamente, almeno durante le prime lezioni, rispettando l'accento di ogni singola parola. Questi meccanismi comunque si attivano ogni qual volta che introduciamo una nuova parola, anche se il discente padroneggia bene la lingua straniera. Il nuovo termine sarà compreso meglio se aiutiamo l'alunno a reperire una parola in rima. In riferimento all'età invece, ricordiamoci che gli adulti hanno bisogno di più tempo e maggiori energie rispetto i bambini, quindi bisognerà dedicare più tempo per questa fase.

Una volta che è stato individuato il formato fonologico avviene la selezione lessicale, che si avvia in automatico per gli individui madrelingua, grazie l'ampio bagaglio lessicale che possiedono infatti, identificano la parola senza nessuna difficoltà $\mathrm{e}$ in breve tempo. Gli studenti invece che vogliono comprendere una nuova parola in lingua straniera eseguono un transfer dalla L2 alla L1, se come già detto, il livello di padronanza è basso e/o hanno un'età superiore ai sette anni. Abbiamo descritto nel paragrafo precedente che, in questa fase, prima si recuperano le parole con lo stesso suono finale e dopo si seleziona quella più appropriata (modello della coorte). L'insegnante quindi può proporre alcune famiglie di parole con questa caratteristica, magari aiutandosi con un rimario. I vocaboli scelti devono essere ripetuti per più lezioni, per essere immagazzinati. Inizialmente la comprensione è facilitata se abbiniamo una traduzione, che può essere proposta verbalmente, attraverso la lingua 
madre, oppure usando il codice iconico, dove alla parola pronunciata si abbina un disegno che rappresenti il significato. Sono fondamentali anche le informazioni contestuali, che come abbiamo già detto, possono essere espresse con i gesti, il tono di voce, ma si potrebbero fornire anche servendosi di qualche piccolo oggetto da esibire all'inizio della lezione in modo da anticipare il gruppo di parole che verranno presentate successivamente.

La comprensione orale del messaggio è completata quando si individuano le informazioni morfosintattiche della parola. Questa funzione è assolta dalla memoria esplicita, altro tipo di memoria a lungo termine caratterizzata da: volontà, focalizzazione dell'attenzione, consapevolezza, verbalizzazione, apprendimento veloce (CANGIÀ 2011, p. 138; DALOISO 2009, p. 70). Si consiglia dunque di adoperare sempre gli stessi vocaboli per proporre agli studenti una riflessione grammaticale. L'analisi delle parole prevede un apprendimento, a differenza della fonetica che viene acquisita inconsapevolmente, l'alunno quindi deve essere incoraggiato dall'insegnante. La motivazione, come afferma Balboni (2008, pp. 33-35), è fondamentale per imparare espressioni in lingua straniera e ancor di più in contesto estero. Lo studente, infatti, è esposto alla nuova lingua solo per poche ore settimanali, quindi l'insegnante dovrebbe il più possibile rendere piacevole la lezione. Si consiglia perciò di coinvolgere gli alunni nella scelta dell'argomento, che potrebbe riguardare i loro passatempi preferiti, oppure interessi di tipo professionale. Riteniamo che se presentiamo parole in rima baciata l'analisi grammaticale risulti più semplice, come anche la memorizzazione delle eccezioni.

\begin{tabular}{|c|c|}
\hline Fasi & Implicazioni glottodidattiche \\
\hline Analisi uditiva & Predisporre un ambiente di apprendimento che orienti \\
\hline
\end{tabular}




\begin{tabular}{|l|l|}
\hline $\begin{array}{l}\text { Trasformazione dello } \\
\text { stimolo acustico in } \\
\text { formato fonologico }\end{array}$ & $\begin{array}{l}\text { Marcare l'intonazione; porre attenzione agli aspetti } \\
\text { emotivi che suscitiamo negli alunni, scandire le parole } \\
\text { lentamente. } \\
\text { Metodo induttivo }\end{array}$ \\
\hline Selezione lessicale & $\begin{array}{l}\text { Proporre parole in rima baciata; tradurre le parole } \\
\text { italiane servendo della L1, immagini, oggetti, gesti, ecc. }\end{array}$ \\
\hline $\begin{array}{l}\text { Accesso al lemma e } \\
\text { unificazione } \\
\text { grammaticale }\end{array}$ & $\begin{array}{l}\text { Riflessione grammaticale sulle parole precedentemente } \\
\text { presentate; sostenere la motivazione grazie l'utilizzo di } \\
\text { strumenti accattivanti e argomenti che interessino gli } \\
\text { alunni. }\end{array}$ \\
\hline
\end{tabular}

Tabella 2: Fasi della comprensione del messaggio orale e relative implicazioni didattiche.

\section{La produzione del messaggio orale}

In questo paragrafo descriveremo la produzione del messaggio orale facendo riferimento alle nozioni di neurolinguistica e all'apprendimento della lingua italiana all'estero, come abbiamo svolto per il paragrafo 2. Riprendiamo dunque le fasi che propone Marini (2010, pp.101-106) in riferimento a questo processo.

a) Pianificazione concettuale del messaggio. In questa fase il locutore pianifica il messaggio stabilendo un'intenzione comunicativa. In relazione all'enunciato ascoltato (se si tratta di una risposta), o al proprio desiderio di comunicare, il parlante stabilisce cosa dire tenendo in considerazione anche il contesto extralinguistico (situazione, ambiente, ecc.) e le aspettative dell'interlocutore. Il pensiero inizialmente si focalizza sull'oggetto del messaggio e su l'ordine da rispettare durante l'esposizione e quindi si crea uno schema concettuale (fase di macropianificazione). Successivamente l'informazione viene convertita in forma proposizionale e si attivano $\mathrm{i}$ rispettivi concetti lessicali corrispondenti. Ci chiediamo quale sia il rapporto tra il lessico della 
lingua materna, il lessico della nuova lingua e il sistema concettuale. Recenti studi dimostrano l'esistenza di un sistema lessicale-concettuale tripartito in un magazzino per il lessico della lingua nativa, uno per la seconda lingua e infine un unico sistema concettuale. Il bilingue precoce (vedi paragrafo 1) passa dal sistema concettuale al lessico della lingua straniera senza operare una traduzione. I bilingui tardivi invece, per produrre (ma anche comprendere) un messaggio attingono sempre dal sistema lessicale della lingua madre, che media tra sistema concettuale e lingua straniera.

b) Fase di codifica grammaticale. Recuperati i concetti lessicali il nostro sistema cognitivo ricorre al lessico mentale e più nel dettaglio al lessico fonologico di input dove sono immagazzinate le configurazioni acustiche. Questa fase viene ulteriormente suddivisa in:

i) selezione lessicale: si svolge attraverso un meccanismo prima di attivazione e poi di inibizione, regolato dalla soglia di attivazione che è bassa quando le parole che si vogliono produrre sono usate frequentemente oppure quando non è trascorso molto tempo dall'ultima volta che si è utilizzata, viceversa è alta quando il termine non è espresso abitualmente $\mathrm{o}$ non è stato pronunciato recentemente. Per quanto riguarda l'inibizione invece, il vocabolo viene selezionato tra quelli dello stesso campo semantico, grazie l'alta soglia di attivazione che esclude le parole con lo stesso significato. Per apprendere una nuova espressione, quindi deduciamo che bisogna fornire una grande quantità di stimoli.;

ii) accesso lessicale: si identifica nel lemma le informazioni morfologiche, semantiche e morfosintattiche;

iii) organizzazione frasale: prevede un "livello funzionale" e un "livello 
posizionale": dalla parola selezionata si generano altre parole che vengono disposte in base a relazioni grammaticali specifiche.

c) Codifica morfononologica. Nella fase precedente l'accesso lessicale è seguito dall'accesso al lemma. La produzione del discorso orale prosegue con l'individuazione del lessema, da cui si traggono le nozioni prosodiche e fonologiche, attraverso un processo di sillabazione.

d) Codifica fonetica. I fonemi vengono riconosciuti grazie dei picchi di intensità che caratterizzano le sillabe. È importante osservare che l'italiano contiene più di 12.000 sillabe ed è impossibile pensare che le varie combinazioni siano immagazzinate in modo specifico. I parlanti di madrelingua italiana infatti, usano non più di 80 sillabe ad alta frequenza.

e) Articolazione. Una volta recuperate le sillabe fonologiche si esprime il proprio pensiero riproducendo i suoni, grazie una sequenza di movimenti articolatori.

La produzione, al contrario della comprensione, si sviluppa dal globale al particolare, capiamo in questo modo la circolarità che lega questi due processi.

\subsection{Implicazioni glottodidattiche}

Durante il processo di apprendimento dunque comprensione e produzione di un enunciato sono strettamente collegati, ma in questo paragrafo proponiamo alcune implicazioni glottodidattiche specifiche in quanto la produzione di un messaggio orale differisce per certi aspetti dalla comprensione.

Nella prima fase di produzione del messaggio orale (pianificazione concettuale) deve essere chiaro il contesto, cioè l'argomento e gli scopi 
dell'interlocutore, che a scuola sono gli obiettivi didattici e le aspettative degli alunni. Quando si instaura una relazione comunicativa, l'insegnante oltre che far emergere il nucleo concettuale dovrà scegliere un tema che interessi gli alunni, per coinvolgerli maggiormente durante la lezione, come già detto nel paragrafo 2.1. Per sostenere la motivazione inoltre dovremmo esplicitare chiaramente i nostri scopi. Come scritto nel paragrafo 3, la produzione del messaggio orale si basa molto sulle attese dell'insegnante, quindi gli alunni, consapevoli della loro meta, potranno potenziare in questo modo anche la competenza glottomatetica, che riguarda la capacità di riflettere e prendere consapevolezza sui processi messi in atto per apprendere una nuova lingua. A fine lezione gli studenti potranno chiedersi se il traguardo è stato raggiunto e capire in caso negativo, il motivo del loro insuccesso. In secondo luogo, la fase di pianificazione del messaggio orale può essere facilitata se indichiamo l'ordine da seguire in modo che l'alunno focalizzi l'attenzione sui contenuti. È importante ricordare infine, che gli alunni con un basso livello di padronanza, hanno la necessità di eseguire questa fase prima nella loro lingua nativa. L'insegnante deve essere consapevole quindi che durante la produzione l'alunno farà degli errori dovuti alla traduzione. Dobbiamo quindi tenere in considerazione che l'apprendimento linguistico avviene in un continuum, da L1 a L2 e da L2 a L1 e che quindi gli errori tipici dell'interferenza tra lingue, sono un indice di apprendimento e non di regressione (MAZZOTTA in SANTIPOLO, 2006, p. 55).

Per sviluppare la fase di codifica grammaticale proponiamo di chiedere agli alunni di esporre prima un iperonimo (come per esempio "fiore") e successivamente i rispettivi iponimi (come per esempio "rosa", "iris", "margherita", ecc.). Individuate le parole, il nostro cervello in automatico esegue una rapidissima riflessione grammaticale, per scegliere le parole 
corrette da affiancare. Durante l'apprendimento di una nuova lingua questo processo deve essere guidato, perché come sappiamo, se traduciamo la nostra lingua parola per parola, il significato globale potrebbe essere compromesso. 亡̀ necessario quindi chiedere agli alunni di svolgere un'analisi morfologia (per esempio, se prendiamo in considerazione la parola "rosa", diciamo che è un nome, singolare, femminile), un'analisi semantica (è un fiore) e infine morfosintattica (ad esempio pensare a quale articolo debba essere abbinata e a quali verbi associare). Le parole individuate inizialmente, potranno essere utilizzate infine per produrre un discorso.

Segue la fase di codifica morfofonologica e fonetica, che prevede il recupero delle informazioni prosodiche e fonologiche. Lo studente mentalmente prova a scandire in sillabe le parole scelte e già ordinate mentalmente, ma non sempre il suono può corrispondere a quello udito. La pronuncia non sarà mai identica a quella di un madrelingua, soprattutto se acquisita nell'età adulta, nonostante ciò, per garantire la comprensione, dobbiamo valutare quali errori correggere.

La produzione del messaggio orale può essere favorita anche con il supporto di altri codici, perché ogni studente possiede un canale preferenziale mediante il quale recepisce il messaggio (TORRESAN, 2010, pp. 20-21). Proponiamo quindi di avvalersi anche di disegni, fotografie, senza escludere le tecnologie, che costituiscono un ottimo strumento per l'apprendimento di una nuova lingua. In particolare per il consolidamento della produzione orale si potrebbe sfruttare Skype, per dialogare direttamente con un coetaneo madrelingua.

La seguente tabella sintetizza le fasi e le relative implicazioni glottodidattiche, che un insegnante può scegliere di applicare durante il potenziamento della produzione orale. 


\begin{tabular}{|l|l|}
\hline Fasi & Implicazioni glottodidattiche \\
\hline $\begin{array}{l}\text { Pianificazione } \\
\text { concettuale del } \\
\text { messaggio }\end{array}$ & $\begin{array}{l}\text { Scegliere l'argomento in base interessi degli alunni; } \\
\text { esplicitare gli obiettivi didattici; predisporre una } \\
\text { scaletta per facilitare l'esposizione di un discorso. }\end{array}$ \\
\hline Codifica grammaticale & $\begin{array}{l}\text { Individuazione iperonimo e rispettivi iponimi; analisi } \\
\text { dei verbi. }\end{array}$ \\
\hline $\begin{array}{l}\text { Codifica } \\
\text { morfofonologica e } \\
\text { fonetica }\end{array}$ & $\begin{array}{l}\text { Valutazione dell'errore fonologico: solo se } \\
\text { compromette notevolmente la comprensione. }\end{array}$ \\
\hline
\end{tabular}

Tabella 2:Fasi della produzione del messaggio orale e relative implicazioni glottodidattiche.

\section{L'Unità di Apprendimento per sviluppare la competenza comunicativa orale}

L'unità di apprendimento è uno strumento che serve all'insegnante per progettare il proprio intervento didattico. Può essere utilizzata anche per una sola lezione infatti, dovrebbe essere breve ed indipendente dalle altre, che compongono l'unità didattica. Il primo passo prima di predisporre l'unità di apprendimento è conoscere gli alunni: età, livello di padronanza della lingua, interessi, ecc. È a partire proprio da queste informazioni che dovremmo individuare un argomento che faccia da sfondo alla nostra unità di apprendimento.

In questo paragrafo riproponiamo le fasi precedentemente descritte, della comprensione e produzione del messaggio orale, alle quali abbineremo la competenza che secondo noi è maggiormente coinvolta e le rispettive tecniche glottodidattiche che si possono utilizzare per svilupparla.

\section{Analisi uditiva}

Si identificano i suoni della propria lingua, distinguendoli da altre fonti sonore o da altre lingue. La competenza fonetica perciò può essere sviluppata 
con l'ausilio di una canzone, oppure in un livello più avanzato, proponendo brani in altre varietà, come per esempio scegliendo un dialetto. La canzone permette agli studenti di aumentare la motivazione, grazie l'intrinseca piacevolezza della musica. Lo stesso obiettivo è raggiunto con l'ascolto di un brano in dialetto, in quanto potrebbe essere interpretato come una piacevole sfida.

\section{Formazione del formato fonologico}

La fonologia a differenza della fonetica si occupa di determinare la funzione dei suoni. La tecnica delle coppie minime consente allo studente di comprendere e analizzare il significato di una parola a partire da un cambiamento di un fonema (per esempio cása e cosa).

\section{Selerione lessicale}

Le parole vengono riordinate in base il medesimo suono iniziale o finale, tale competenza morfofonologica, prevede la conoscenza di una bagaglio di parole da cui se ne posso scegliere una. Si consiglia quindi di proporre, inizialmente, parole in rima.

\section{Unificazione grammaticale}

Individuata la parola si determinano le relazioni con altre parole. Si tratta della competenza morfosintattica che unisce il contesto situazionale con quello verbale per attribuire un significato all'intera frase. Si propone di analizzare dal punto di vista grammaticale parole e verbi e le loro relazioni. In questa fase pertanto l'alunno, attraverso la riflessione, giunge al significato globale.

\section{Pianificazione}

La prima fase di produzione del messaggio orale implica la competenza pragmatica, cioè l'individuazione del contesto situazionale e verbale. L'insegnante quindi dovrebbe esplicitare sia le informazioni inerenti 
l'argomento sia quelle extralinguistiche, che possono essere veicolate grazie l'utilizzo di altri codici, oltre quello verbale. Durante la pianificazione dovrebbe essere sostenuta la motivazione, proprio grazie il coinvolgimento di tutte le modalità sensoriali (visivo, auditivo, cinestesico). In questo modo si aiuta lo studente a cogliere il senso globale del discorso che vuole produrre.

\section{Codifica grammaticale}

Si elabora la struttura delle frasi, capacità tipica della competenza sintattica, che può essere consolidata grazie la produzione di iperonimi, iponimi e il rispettivo collegamento con verbi. L'alunno analizza nel dettaglio le parole per configurarle con una giusta disposizione.

\section{Codifica morfofonologica}

Mentalmente si scompone la parola in sillabe prima di pronunciarla, meccanismo che occupa più tempo quando vogliamo pronunciare una parola troppo lunga, o appena sentita. Il discente perciò reimpiega gli schemi messi in atto durante la comprensione, coinvolgendo però anche l'apparato vocale. Se l'alunno compie spesso errori di pronuncia consigliamo la tecnica della ripetizione.

Proponiamo uno schema di Unità di Apprendimento per lo sviluppo della competenza comunicativa orale (tabella 3).

\begin{tabular}{|l|l|l|l|}
\hline $\begin{array}{l}\text { Processo di } \\
\text { apprendimento }\end{array}$ & Fasi & Competenza & Tecnica \\
\hline Comprensione & Analisi uditiva & Fonetica & Ascolto canzone \\
\cline { 2 - 4 } & $\begin{array}{l}\text { Formazione formato } \\
\text { fonologico }\end{array}$ & Fonologica & Coppie minime \\
\cline { 2 - 4 } & Selezione lessicale & Morfofonologica & Parole in rima \\
\cline { 2 - 4 } & $\begin{array}{l}\text { Unificazione } \\
\text { grammaticale }\end{array}$ & Morfosintattica & $\begin{array}{l}\text { Individuazione } \\
\text { verbi; } \\
\text { relazioni } \\
\text { grammaticali parole }\end{array}$ \\
\hline
\end{tabular}


Lo Sviluppo della Competenza Comunicativa Orale dell'Italiano come Lingua Straniera.

Accenni neurolinguistici e glottodidattici. Martina Brazzolotto

\begin{tabular}{|l|l|l|l|}
\hline Produzione & Pianificazione & Pragmatica & $\begin{array}{l}\text { Esplicitare contesto } \\
\text { situazionale e } \\
\text { verbale (cotesto) }\end{array}$ \\
\cline { 2 - 3 } & $\begin{array}{l}\text { Codifica } \\
\text { grammaticale }\end{array}$ & Sintattica & $\begin{array}{l}\text { Iperonimo-iponimo } \\
\text { analisi verbi }\end{array}$ \\
\cline { 2 - 4 } & $\begin{array}{l}\text { Codifica } \\
\text { morfofonologica }\end{array}$ & Morfofonologica & $\begin{array}{l}\text { Suddivisione in } \\
\text { sillabe; ripetizione }\end{array}$ \\
\hline
\end{tabular}

Tabella 3: Unità di Apprendimento per lo sviluppo della competenza comunicativa orale in classe.

\section{Conclusione}

Le recenti scoperte neurolinguistiche ci permettono di adottare in classe delle tecniche glottodidattiche che siano in linea con la naturale progressione delle fasi di comprensione e produzione del messaggio orale. Questi due processi sono collegati da un passaggio in comune, la comprensione globale, ma ricordiamoci che differiscono l'uno dall'altro, in quanto il primo inizia con l'analisi delle singole parole, mentre per il secondo rappresenta l'ultimo passaggio. Per questi motivi, quando vogliamo sviluppare la comprensione dovremmo iniziare la nostra attività didattica a partire dalle singole parole per arrivare al significato generale, mentre quando vogliamo focalizzarci sulla produzione dovremmo indirizzare gli studenti prima sul significato globale e successivamente sulle singole parole.

Per quanto riguarda le indicazioni generali, concludiamo dicendo che l'insegnante dovrebbe facilitare l'apprendimento attraverso delle modalità che sostengano lo studente, che dovrà sperimentare prevalentemente situazioni di successo scolastico. L'insegnamento dunque sarà di alta qualità quando ci chiediamo la motivazione dell'insuccesso dei nostri alunni. Solo in quel momento capiremo che coinvolgere emotivamente, sviluppare la competenza glottomatetica e variare le lezioni, usando tutti i codici linguistici possibili, 
sono metodi che garantiscono l'apprendimento linguistico.

\section{Riferimenti bibliografici}

BALBONI, Paolo E. Le sfide di Babele: Insegnare le lingue nelle società complesse. Nuova edizione. Torino: Utet Università, 2008.

BEAR, Mark F.; CONNORS, Barry W.; PARADISO, Michael A. Neuroscien₹e: Esplorando il cervello. Terza edizione. Milano: Elsevier, 2009.

CANGIÀ, Caterina. Lingue altre 1: Conoscerle e coltivarle. Brescia: La Scuola, 2011.

DALOISO, Michele. I fondamenti neuropsicologici dell'educazione linguistica. Venezia: Cafoscarina, 2009.

FABBRO, Franco. Il cervello bilingue: Neurolinguistica e poliglossia. Roma: Astrolabio, 1996.

. Neuropedagogia delle lingue: Come insegnare le lingue ai bambini. Roma: Astrolabio, 2004.

FERRARI, Marcella; PALLADINO, Paola. L'apprendimento della lingua straniera. Roma: Carocci, 2012.

KRASHEN, Stephen D. The Input Hypothesis: Issues and Implications. New York: Longman, 1985.

LIBERMAN, Alvin M.; MATTINGLY, Ignatius G. The Motor Theory of Speech Perception Revised. Cognition, v. 21, 1985, pp. 1-36.

MARINI, Andrea. Manuale di neurolinguistica: Fondamenti teorici, tecniche di indagine, applicazioni. Roma: Carocci, 2010.

MARSLEN-WILSON, William. Functional Parallelism in Spoken Word Revista Italiano UERJ Vol. $4-\mathrm{n}^{\circ} 4-2013$ p. 81 
Lo Sviluppo della Competenza Comunicativa Orale dell'Italiano come Lingua Straniera. Accenni neurolinguistici e glottodidattici. Martina Brazzolotto

Recognition. Cognition, v. 25, 1987, pp. 71-102.

MAZZOTTA, Patrizia. La dimensione cognitiva dell'apprendimento dell'italiano come lingua seconda. In SANTIPOLO, Matteo. L'italiano: Contesti di insegnamento in Italia e all'estero. Torino: Utet Università, 2006, 49-74.

PERANI, Daniela. Brain processing of native and foreign languages. Neuroreport, v. 7, 1996, 2439-2444.

SANTIPOLO, Matteo. L'italiano: Contesti di insegnamento in Italia e all'estero. Torino: Utet Università, 2006.

TORRESAN, Paolo. The Theory of Multiple Intelligences and Language Teaching. Perugia: Guerra, 2010. 